\title{
Prevalence and Prognostic Implications of PD-L1 Expression in Soft Tissue Sarcomas
}

\author{
Mohamed Kelany ${ }^{1 *}$, Thomas FE. Barth ${ }^{2}$, Dina Salem ${ }^{1}$ and Marwa M. Shakweer ${ }^{3,4}$ \\ ${ }^{1}$ Department of Clinical Oncology, Ain Shams University, Cairo, Egypt, ${ }^{2}$ Department of Pathology, Ulm University, Ulm, Germany, \\ ${ }^{3}$ Department of Pathology, Ain Shams University, Cairo, Egypt, ${ }^{4}$ Department of Pathology, Badr University in Cairo (BUC), Cairo, Egypt
}

Background: PD-L1 expression differs from 19 to $92 \%$ in various cancer subtypes. Its expression carries a worse prognostic value in various malignancies and could also be used as a predictive marker for immune checkpoint inhibitor response. This study aimed to explore the prevalence of PD-L1 expression in soft tissue sarcomas and the correlation of PD-L1 expression with clinicopathological features.

Patients and Methods: The tissue samples of 50 patients with STS were tested for PDL1 expression using immunohistochemistry (IHC). We followed a 6-step proportional scoring system. The patients were treated at Ain Shams University Hospital from 2011 to 2017. We also explored the correlation of PD-L1 expression with different clinical features of the patients. The chi-square test was used to calculate the differences among variables.

Results: Twelve cases (24\%) showed positive PD-L1 expression with the highest prevalence in rhabdomyosarcoma and desmoid tumors $(2 / 2$ and $2 / 3$ cases, respectively), followed by GIST in 2/4 cases and liposarcoma in 3/11 cases. Patients with positive PD-L1 expression showed a trend for worse survival, with a median overall survival of 11 months vs. 19 months for patients with negative PD-L1 expression ( $p$-value $=0.1$ ) and a mean PFS of 6 months vs. 11 months for patients with negative PD-L1 expression $(p$-value $=0.1)$. However, these findings did not reach statistical significance.

OPEN ACCESS

Edited by: József Tímár

Semmelweis University, Hungary

*Correspondence: Mohamed Kelany kelaney.m@med.asu.edu.eg

Received: 10 March 2021 Accepted: 09 June 2021 Published: 01 July 2021

Citation: Kelany M, Barth TFE, Salem D and Shakweer MM (2021) Prevalence and Prognostic Implications of PD-L1 Expression in Soft Tissue Sarcomas. Pathol. Oncol. Res. 27:1609804. doi: 10.3389/pore.2021.1609804
Conclusion: Although the results did not reach statistical significance due to the small number of cases, PD-L1 expression could represent a prognostic factor for poor outcome. Larger clinical trials are recommended for the validation of PD-L1 as a poor prognostic biomarker.

Keywords: biomarker, prognosis, PD-L1 expression, soft tissue sarcomas, prevalence

\section{INTRODUCTION}

Soft-tissue sarcoma is a challenging disease area because it is a heterogeneous disease with over 50 different subtypes (1). Second, it is a rare disease that accounts for over $20 \%$ of all pediatric solid malignant cancers and less than $1 \%$ of all adult solid malignant cancers (2).

Tumor initiation, progression, and responses to therapy are highly related to the tumor microenvironment, including cells and molecules of the immune system $(3,4)$.

The expression rate of PD-L1 in human malignant tumors has been reported to vary from 19 to $92 \%$ and is associated with the progression and poor prognosis of various human cancers. In 
TABLE 1 | Cologne score.

Table of cologne score
Category
Cut off

Interval

0
$<1 \%$
$0-1 \%$

1

$\geq 1 \%$

$\geq 1 \%$

$<5 \%$

$\begin{array}{cc}1 & 2 \\ \% & \geq 5 \% \\ \% & \geq 5 \% \\ \% & <10 \%\end{array}$

3
$\geq 10 \%$
$\geq 10 \%$
$<25 \%$

$\begin{aligned} & 4 \\ \geq & 25 \% \\ \geq & 25 \% \\ < & 50 \%\end{aligned}$

addition, intratumor infiltration of PD1-positive T-cells was positively correlated with the progression of human malignant tumors (5).

PD-L1 expression is rarely studied in soft tissue sarcoma. In one study that analyzed PD-L1 expression in 82 STS patients, PDL1 expression was identified in $43 \%$ of STS patients and considered an independent adverse prognostic factor for the overall survival of STS patients (6).

We evaluated the expression of PD-L1 in soft tissue sarcoma specimens on paraffin-embedded tissue by immunohistochemistry. Our primary endpoint was the detection of the prevalence of PD-L1 expression in soft tissue sarcoma and its correlation with the clinicopathological features of the tumor. The secondary endpoint was the prognostic significance of PD-L1 in soft tissue sarcoma.

\section{Sample Size Justification}

The necessary sample size was calculated using PASS Sample Size Software, setting the type- 1 error $(\alpha)$ at 0.05 (95\% confidence interval). The power $(1-\beta)$ at 0.8 was based on a previous study (7), which showed that PD-L1 expression was $19 \%$ in soft tissue sarcoma tumors collectively. The calculation according to these values produced a minimal sample size of 50 cases.

\section{METHODS}

This study comprised a total of 50 specimens of surgically removed, formalin-fixed, and paraffin-embedded tumors. Cases were retrieved from the archives of Ain Shams University Hospital during the period from 2011 to 2017 in Cairo, Egypt.

Cases with adequate paraffin tissue samples were included in this study. The study was carried out with full local ethics approval. Various types of soft tissue sarcomas were included. Tumors were classified according to $\mathrm{WHO} / 2013$ pathological classification (fourth edition) (8), including desmoid and GIST and excluding bone sarcomas.

We reviewed the medical records for patient data, including age, race, tumor location, histological type and grade, tumor stage based on the seventh edition of the American Joint Committee on Cancer guideline of tumor-node-metastasis (TNM) classification, and resection margin status for resected tumors.

\section{Immunohistochemical Staining}

Immunohistochemistry (IHC) staining was performed on paraffin-embedded tissue sections with a labeled streptavidin-a biotin-peroxidase complex technique using an anti-PD-L1 antibody (CD274 molecule; catalog number: Cell Signaling 13,684, dilution: 1/200). Antigens of PD-L1 were retrieved by microwaving the sections in citrate buffer for $20 \mathrm{~min}$. The final reaction product was developed with diaminobenzidine.

\section{Immunohistochemical Analysis}

We followed a 6-step scoring system (Cologne Score) for the assessment of PD-L1 positivity in sarcomas (Table 1). Only tumor cells with membranous positivity were considered positive for PD-L1. Cytoplasmic positivity was disregarded. Tumor cells were quantified by evaluating the ratio of stained and unstained cells (number of PD-L1-positive tumor cells/ number of all tumor cells) $(9,10)$.

\section{RESULTS}

The cohort consisted of 27 (54\%) female and 23 (46\%) male patients with a median age of 42.5 years (range 18-79). Most tumors were of grade G2 (34\%) and G3 (46\%). The majority of the patients did not have metastases at presentation (92\%). The most frequent histologic subtypes were liposarcoma (22\%), followed by leiomyosarcoma (18\%) and ULP (15\%). Eighty-four percent (42 patients) underwent surgical treatment, with R0 margins in $38 \%$ of these patients. Thirty percent of patients developed metastases, with the lung as the predominant site (see Table 2).

\section{PD-L1 Expression in Tumor Specimens and Its Clinical Correlations}

We defined PD-L1 expression as positive if the Cologne Score equaled three or more, as defined elsewhere (Figure 1; 15). Tumor cell PD-L1 expression was observed in 12 (24\%) cases. Of all sarcoma subtypes sampled, the highest prevalence of PD-L1 expression was noted in rhabdomyosarcoma (2/2 cases) and desmoid tumors ( $2 / 3$ cases), followed by GIST ( $2 / 4$ cases) and liposarcoma (3/11 cases). The median OS of patients with positive PD-L1 expression (Cologne Score $\geq 3$ ) was 11 months, while it was 19 months for patients with negative PD-L1 expression [hazard ratio $(\mathrm{HR})=3.086,95 \%$ confidence interval $(\mathrm{CI})=0.59-16.01, p$-value $=0.14]$. The mean PFS of patients with positive PD-L1 expression was 6 months, while it was 10.6 months for patients with negative PD-L1 status [hazard ratio $(\mathrm{HR})=1.806,95 \%$ confidence interval $(\mathrm{CI})$ $=0.55-5.9, p$ value $=0.28]$ (Figure 2$)$.

\section{DISCUSSION}

In this study, we evaluated the expression of PD-L1 in various sarcoma subtypes and explored its correlations with different clinicopathological features and survival. PD-L1 expression in 
TABLE 2 | Summary of patient characteristics and clinicopathological features.

\begin{tabular}{l} 
Grade \\
\hline FNCLCC: \\
G1 \\
G2 \\
G3 \\
GIST: \\
Low \\
High \\
Surgery \\
Yes \\
No \\
Margin \\
NA \\
R0 \\
R1 \\
R2 \\
Adjuvant treatment \\
Chemotherapy: \\
Yes \\
No \\
Radiotherapy: \\
Yes \\
No \\
Recurrence \\
Local and nodal \\
Systemic \\
1st Line chemotherapy in systemic recurrence \\
Anthracycline: \\
Single agent \\
Combination \\
TKl: gleevec \\
Response to the 1st line treatment \\
PD \\
PR \\
SD \\
\\
No \\
No
\end{tabular}

\section{Patient characteristics}

Age median (range)

Sex

Female

Male

Histology

Angiosarcoma

Desmoid

Epithelioid

Fibrosarcoma (adu.)

(Myxoid)

GIST

Histiocytic sarcoma

Leiomyosarcoma

Liposarcoma (ULP)

(deDIF.)

(Myxoid)

(ULP)

Pleomorphic sarcoma (UPL)

Rhabdomyosarcoma

Synovial

T Stage

$\mathrm{T} 1$

$\mathrm{T} 2$

T3

T4

$M$ Stage

MO

M1
N (\%)

6 (12)

$17(34)$

$23(46)$

$3(6)$

1 (2)

$42(84)$

8 (16)

8 (16)

19 (38)

19 (38)

4 (8)

37 (74)

$13(26)$

28 (56)

22 (44)

15 (32.6)

14 (30.6)

7 (14)

$15(30)$

1 (2)

$14(60.9)$

$6(26.1)$

3 (13)

N (\%)

42.5 (18-79)

27 (54)

23 (46)

1 (2)

3 (6)

2 (4)

2 (4)

4 (8)

4 (8)

1 (2)

9 (18)

$1(2)$

1 (2)

4 (8)

5 (10)

8 (16)

2 (4)

3 (6)

4 (8)

$17(34)$

21 (42)

8 (16)

46 (92)

4 (8)

(Continued in next column)
TABLE 2 | (Continued) Summary of patient characteristics and clinicopathological features.

\begin{tabular}{lc}
\hline Patient characteristics & N (\%) \\
\hline Original location & \\
Abdomen/pelvis/R & $18(36)$ \\
Extremities & $26(52)$ \\
Head and neck & $2(4)$ \\
Testicular & $1(2)$ \\
Trunk & $3(6)$
\end{tabular}

tumor cells was observed in 12 (24\%) of 50 soft tissue sarcoma cases of different subtypes. The tumor cell PD-L1 expression patterns vary in the literature (7), i.e., reports of positive expression in $12 \%$ of tumor cells vs. reports that described positive expression in $48 \%$ and $65 \%(11,14)$. Table 3 summarized the most relevant data published in this area.

This discrepancy of the published data may be related to the lack of harmonization of the PD-L1 assay as well as the use of different antibody types and different definitions of a positive result with a specific cut-off. The second reason is related to soft tissue sarcoma itself, as it is not one disease but a heterogeneous group of diseases. We report here a significant variation in PD-L1 expression based on the histological subtypes $(p=0.018)$. This finding was also reported by others, who found that the histological subtypes of soft tissue sarcoma were significantly correlated with PD-L1 expression $(p=0.004)(5)$.

Other than the histological subtypes, we did not identify relationships between tumor PD-L1 expression and clinicopathological characteristics. These results are similar to those of (7) and (5) and in contrast to those of (14), who reported that PD-L1 expression was also significantly associated with higher tumor stage, deep-seated sarcoma, distant metastasis, higher histologic grade, tumor differentiation, and tumor necrosis. Furthermore (21), found that PD-L1 expression was significantly associated with high tumor grade and the age of patients with soft tissue sarcomas.

In other solid malignancies, the level of PD-1/PD-L1 expression is considered an independent factor for worse survival. For example, in renal cell carcinoma, the expression of PD-1 in tumor-infiltrating lymphocytes is considered a poor prognostic factor for survival (21). Additionally, in gastric cancer and lung cancer, PD-L1 expression was established as an independent prognostic factor for poor OS $(15,12)$.

We found that soft tissue sarcoma patients with positive PD-L1 expression showed a median OS of 11 months compared with 19 months for patients with negative PD-L1 expression and a mean PFS of 6 months compared with 11 months for patients with negative PD-L1 expression. We consider this finding clinically significant, as the $p$-value was 0.1 for OS and 0.2 for PFS and therefore indicated a trend. These results confirm the data of $(7)$, who reported a median OS of 10.4 months in PD-L1-positive patients vs. not reached in PD-L1-negative patients, with a $p$-value of 0.8 owing to the small sample size, according to the author's interpretation.

In contrast (5), found that the expression of PD-L1 in tumor tissue significantly predicted shortened OS [ 5 years OS rate, $48 \%$ vs. $68 \%$; 

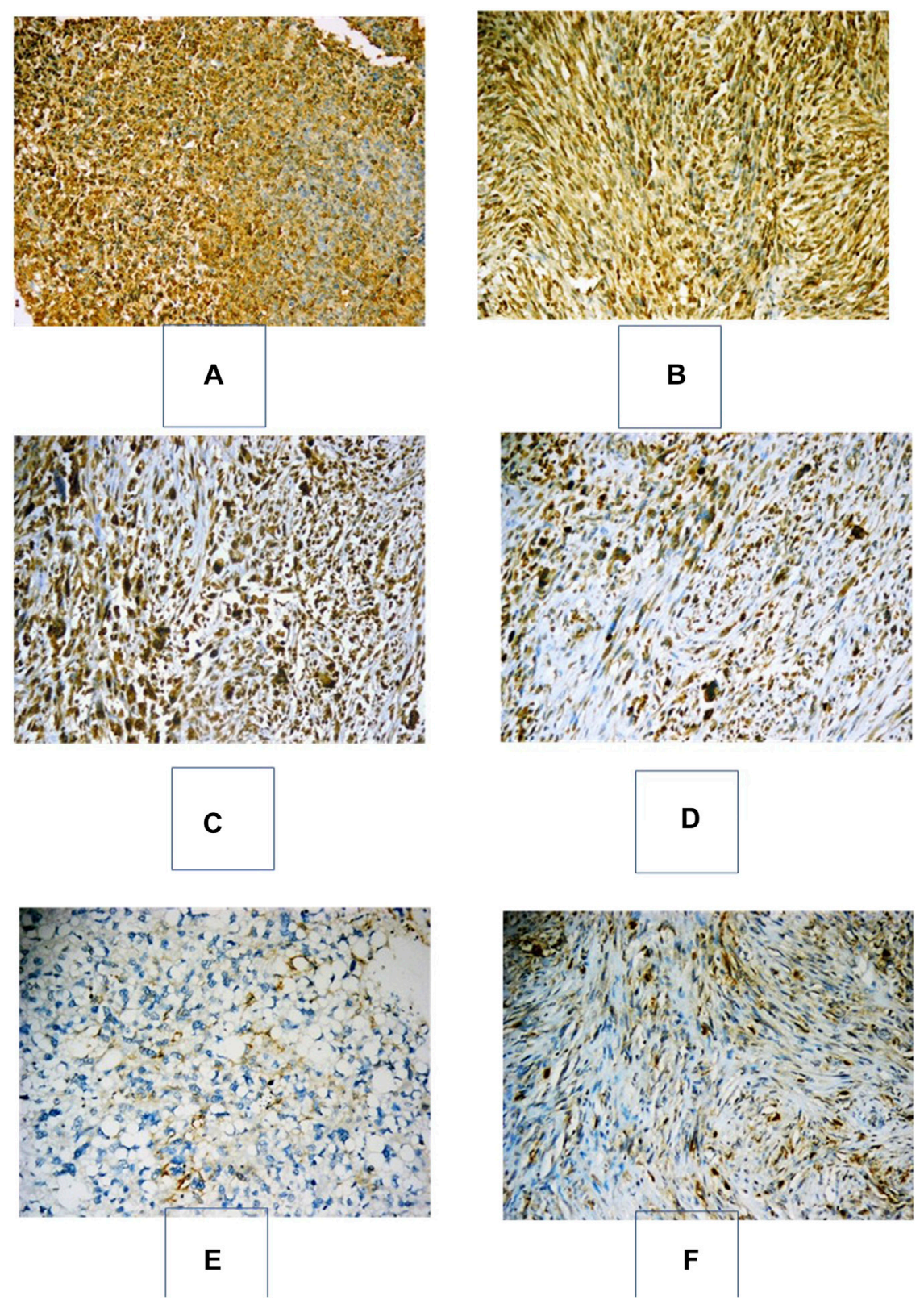

FIGURE 1 | Immunohistochemical expression of PD-L1 in various soft-tissue sarcomas. (A): Embryonal RMS with strong positive expression of PD-L1 in >50\% of neoplastic cells, "Cologne Score 5" (PD-L1x200). (B) GIST with strong positive expression of PD-L1 in >50\% of neoplastic cells, "Cologne Score 5" (PD-L1x200). (C) Pleomorphic undifferentiated sarcoma with strong positive expression of PD-L1 in $>50 \%$ of neoplastic cells, "Cologne Score 5" (PD-L1x200). (D) Pleomorphic undifferentiated sarcoma with moderate positive expression of PD-L1 in 25-50\% of neoplastic cells, "Cologne Score 4" (PD-L1x200). (E): A pleomorphic liposarcoma with positive membranous moderate immunostaining for PD-L1 in 5-10\% of neoplastic cells "Cologne score 2" (PD-L1×200). (F): Fibromatosis with positive membranous moderate immunostaining for PD-L1 in 5-10\% of neoplastic cells "Cologne score 2" (PD-L1×200).

hazard ratio $(\mathrm{HR})=2.545 ; 95 \%$ confidence interval $(\mathrm{CI})=1.16-5.56$; $p=0.015]$ (14). reported that soft tissue sarcoma patients with a PD$1+/ \mathrm{PD}-\mathrm{L} 1+$ phenotype had the shortest survival time and a more progressive STS phenotype. Even in low-stage STS, the five-year survival rate of the PD-1+/PD-L1+ subgroup was only $29 \%$, and the ten-year survival rate was $0 \%$. In contrast, the ten-year survival rates of the PD-1-/PD-L1- subgroups were $94 \%$ in low-stage STS and $67 \%$ in high-stage STS.
The limitations of our analysis are the heterogeneity of the samples, the small sample size, and the imbalanced histological subtypes between the two groups. The study findings have to be confirmed on a larger scale, involving more patients as well as defined larger groups of each subtype.

Additionally, evaluating PD-L1 expression at an isolated time point or at the interval between biopsy and treatment may not represent its true prevalence. Furthermore, the primary disease and 

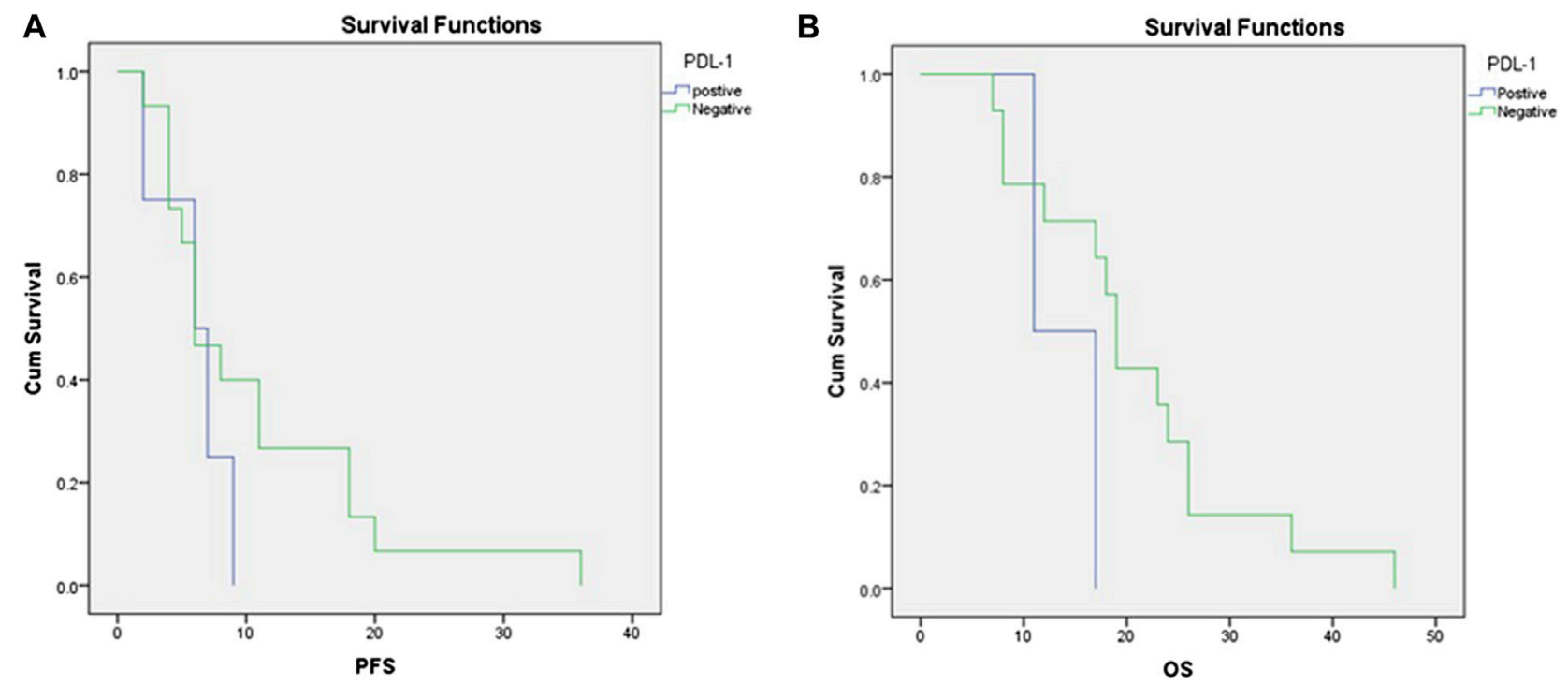

FIGURE 2 | Correlations between PD-L1 expression and (A) progression-free survival and (B) overall survival.

TABLE 3 | Characteristics of the published studies tested the expression of PDL-1 in soft tissue sarcoma.

\begin{tabular}{|c|c|c|c|c|c|}
\hline Study & Year & Pts numbers & Type & Pdl-1 assessment & $\begin{array}{c}\% \text { Of } \\
+ \text { expression }\end{array}$ \\
\hline (11) & 2018 & 128 & Retrospective & $॥ \mathrm{HC}$ & $40.4 \%$ \\
\hline (12) & 2013 & 105 & Retrospective & $\mathrm{IHC}$ & $64.7 \%$ \\
\hline (5) & 2016 & 82 & Retrospective & $\Vdash H C$ & $43 . \%$ \\
\hline (7) & 2015 & 47 & Retrospective & $\mathrm{IHC}$ & $8.5 \%$ \\
\hline (13) & 2015 & 59 & Retrospective & $\mathrm{IHC}$ & $59.3 \%$ \\
\hline (14) & 2017 & 163 & Retrospective & $\mathrm{IHC}$ & $11.7 \%$ \\
\hline (15) & 2018 & 46 & Retrospective & $\mathrm{HC}$ & $45.7 \%$ \\
\hline (16) & 2018 & 81 & Retrospective & $\mathrm{IHC}$ & $59 \%$ \\
\hline (17) & 2017 & 208 & Retrospective & $\mathrm{IHC}$ & $8.65 \%$ \\
\hline (18) & 2016 & 66 & Retrospective & $\mathrm{IHC}$ & $30.3 \%$ \\
\hline (19) & 2017 & 758 & Retrospective & RNA seq & $41 \%$ \\
\hline (20) & 2017 & 162 & Retrospective & RNA seq & $21.1 \%$ \\
\hline
\end{tabular}

the presence of a metastatic site may further impact the value of PDL1 expression, and the analysis in our study involved samples mainly originating from early nonmetastatic stages. However, it is well known that this may reflect failed immune surveillance and tumor escape in advanced and metastatic disease.

\section{DATA AVAILABILITY STATEMENT}

The original contributions presented in the study are included in the article/supplementary material, further inquiries can be directed to the corresponding author.

\section{ETHICS STATEMENT}

The studies involving human participants were reviewed and approved by the Ain Shams University Faculty of Medicine
Research Ethics Committee, FWA 000017585. Written informed consent for participation was not required for this study in accordance with the national legislation and the institutional requirements.

\section{AUTHOR CONTRIBUTIONS}

MK data collection, data analysis, and writing. TB reviewed the staining process and the scoring, writing and reviewing. DS writing and reviewing. MS staining and pathological examination and scoring.

\section{FUNDING}

This work was a part of the Master Online Advanced Oncology Program of Ulm University funded by the ESO European School of Oncology. 


\section{CONFLICT OF INTEREST}

The authors declare that the research was conducted in the absence of any commercial or financial relationships that could be construed as a potential conflict of interest.

\section{REFERENCES}

1. Burningham Z, Hashibe M, Spector L, and Schiffman JD. The Epidemiology of Sarcoma. Clin Sarcoma Res (2012) 2(1):14. doi:10.1186/2045-3329-2-14

2. SEER ${ }^{\star}$ Stat Databases: November 2017 Submission [Internet]. [cited $2021 \mathrm{Feb}$ 15]. Available from: https://seer.cancer.gov/data-software/documentation/ seerstat/nov2017/.

3. Chen DS, and Mellman I. Oncology Meets Immunology: The CancerImmunity Cycle. Immunity (2013) 39(1):1-10. doi:10.1016/ j.immuni.2013.07.012

4. Martin V, Raica M, and Cimpean AM. First-Line Immunophenotyping in the Pathologic Diagnosis of Soft Tissue Tumors. 2004;54(2):122-7.

5. Kim C, Kim EK, Jung H, Chon HJ, Han JW, Shin K-H, et al. Prognostic Implications of PD-L1 Expression in Patients with Soft Tissue Sarcoma. BMC Cancer (2016) 16(1):434. doi:10.1186/s12885-016-2451-6

6. Kim C, Kim EK, Han JW, Chon HJ, Heo SJ, Lee YH, et al. Clinical Pattern and Implication of PD-L1 Expression in Soft-Tissue Sarcoma. Jco (2015) 33:10565. doi:10.1200/jco.2015.33.15_suppl.10565

7. D’Angelo SP, Shoushtari AN, Agaram NP, Kuk LX, Qin L-X, Dickson MA, et al. Prevalence of Tumor-Infiltrating Lymphocytes and PD-L1 Expression in the Soft Tissue Sarcoma Microenvironment. Hum Pathol (2015) 46(3):357-65. doi:10.1016/j.humpath.2014.11.001

8. Jo VY, and Fletcher CDM. WHO Classification of Soft Tissue Tumours: an Update Based on the 2013 (4th) Edition. Pathology (2014) 46(2):95-104. doi:10.1097/PAT.0000000000000050

9. Herbst RS, Baas P, Kim D-W, Felip E, Pérez-Gracia JL, Han J-Y, et al. Pembrolizumab versus Docetaxel for Previously Treated, PD-L1-Positive, Advanced Non-small-cell Lung Cancer (KEYNOTE-010): a Randomised Controlled Trial. Lancet (2016) 387:1540-50. doi:10.1016/S0140-6736(15) 01281-7

10. Scheel AH, Dietel M, Heukamp LC, Jöhrens K, Kirchner T, Reu S, et al. Harmonized PD-L1 Immunohistochemistry for Pulmonary Squamous-Cell and Adenocarcinomas. Mod Pathol (2016) 29(10):1165-72. doi:10.1038/ modpathol.2016.117

11. Boxberg M, Steiger K, Lenze U, Rechl H, von Eisenhart-Rothe R, Wörtler K, et al. PD-L1 and PD-1 and Characterization of Tumor-Infiltrating Lymphocytes in High Grade Sarcomas of Soft Tissue - Prognostic Implications and Rationale for Immunotherapy. OncoImmunology (2018) 7(3). doi:10.1080/2162402x.2017.1389366

12. Kim J, Moon YJ, Kwon KS, Bae JS, Wagle S, Kim KM, et al. Tumor Infiltrating PD1-Positive Lymphocytes and the Expression of PD-L1 Predict Poor Prognosis of Soft Tissue Sarcomas. PLoS ONE (2013) 8(12):1-9. doi:10.1371/journal.pone.0082870

\section{ACKNOWLEDGMENTS}

Special thanks to Uta Schmidt-Straßburger, Scientific Director of this program, for her outstanding support and efforts.

13. Chowdhury F, Dunn S, Mitchell S, Mellows T, Ashton-Key M, and Gray JC. $\mathrm{PD}-\mathrm{L} 1$ and $\mathrm{CD} 8{ }^{+} \mathrm{PD} 1{ }^{+}$Lymphocytes Exist as Targets in the Pediatric Tumor Microenvironment for Immunomodulatory Therapy. OncoImmunology (2015) 4(10). doi:10.1080/2162402x.2015.1029701

14. Que Y, Xiao W, Guan Y, Liang Y, Yan S, Chen H, et al. PD-L1 Expression Is Associated with FOXP3 + Regulatory T-Cell Infiltration of Soft Tissue Sarcoma and Poor Patient Prognosis. J Cancer 2018;8:2018-25. doi:10.7150/jca.18683

15. Patel KR, Martinez A, Stahl JM, Logan SJ, Perricone AJ, Ferris MJ, et al. Increase in PD-L1 Expression after Pre-operative Radiotherapy for Soft Tissue Sarcoma. OncoImmunology (2018) 7(7). doi:10.1080/ 2162402x.2018.1442168

16. Pollack SM, He Q, Yearley JH, Emerson R, Vignali M, Zhang Y, et al. T-cell Infiltration and Clonality Correlate with Programmed Cell Death Protein 1 and Programmed Death-Ligand 1 Expression in Patients with Soft Tissue Sarcomas. Cancer (2017) 123(17). doi:10.1002/cncr.30726

17. van Erp AEM, Versleijen-Jonkers YMH, Hillebrandt-Roeffen MHS, van Houdt L, Gorris MAJ, van Dam LS, et al. Expression and Clinical Association of Programmed Cell Death-1, Programmed Death-Ligand-1 and CD8+ Lymphocytes in Primary Sarcomas Is Subtype Dependent. Oncotarget (2017) 8(41). doi:10.18632/oncotarget.19071

18. Paydas S, Bagir EK, Deveci MA, and Gonlusen G. Clinical and Prognostic Significance of PD-1 and PD-L1 Expression in Sarcomas. Med Oncol (2016) 33(8). doi:10.1007/s12032-016-0807-Z

19. Bertucci F, Finetti P, Perrot D, Leroux A, Collin F, le Cesne A, et al. PDL1 Expression Is a Poor-Prognosis Factor in Soft-Tissue Sarcomas. OncoImmunology (2017) 6(3). doi:10.1080/2162402x.2016.1278100

20. Budczies J, Mechtersheimer G, Denkert C, Klauschen F, Mughal SS, Chudasama P, et al. PD-L1 (CD274) Copy Number Gain, Expression, and Immune Cell Infiltration as Candidate Predictors for Response to Immune Checkpoint Inhibitors in Soft-Tissue Sarcoma. OncoImmunology (2017) 6(3). doi:10.1080/2162402x.2017.1279777

21. Thompson RH, Dong H, Lohse CM, Leibovich BC, Blute ML, Cheville JC, et al. PD-1 Is Expressed by Tumor-Infiltrating Immune Cells and Is Associated with Poor Outcome for Patients with Renal Cell Carcinoma. Clin Cancer Res (2007) 13:1757-61. doi:10.1158/1078-0432.CCR-06-2599

Copyright (C) 2021 Kelany, Barth, Salem and Shakweer. This is an open-access article distributed under the terms of the Creative Commons Attribution License (CC BY). The use, distribution or reproduction in other forums is permitted, provided the original author(s) and the copyright owner(s) are credited and that the original publication in this journal is cited, in accordance with accepted academic practice. No use, distribution or reproduction is permitted which does not comply with these terms. 